\title{
Renforcement d'un remblai par inclusions rigides : approche expérimentale
}

\author{
L. BRIANÇON \\ Conservatoire national \\ des arts et métiers \\ 2, rue Conté \\ 75141 Paris Cedex 03 \\ laurent.briancon@cnam.fr
}

\section{B. SIMON}

Terrasol

Immeuble Central Seine 42-52, quai de la Rapée CS 71230

75583 Paris Cedex 12 b.simon@terrasol.com
Le projet national français ASIRI (Amélioration des Sols par Inclusions RIgides) a été initié pour améliorer les connaissances sur le renforcement des sols par inclusions rigides verticales et proposer des règles de mise en œuvre et de dimensionnement. Dans le cadre de ce projet, une expérimentation en vraie grandeur constituée de quatre plots sous un remblai a été réalisée. Une instrumentation complète a été installée pour mesurer les variations de pression interstitielle dans le sol compressible, le transfert de charge vers les têtes d'inclusions, le tassement des inclusions rigides et du sol compressible, les déplacements latéraux en pied de talus du sol et des têtes d'inclusions et les déformations des nappes géosynthétiques. L'ensemble de ces mesures a permis d'acquérir des données expérimentales de tout premier ordre concernant le comportement des remblais sur inclusions rigides.

Mots-clés : inclusions rigides, remblais, géosynthétiques, instrumentation, renforcement.

\section{Reinforcement of pile-supported embankement: experimental study}

The French national research project ASIRI was launched to improve the knowledge in this field and to draft a document constituting the Guide-lines relating to the set up and the design of embankments and pavements on ground reinforced by rigid piles. In this frame, a full-scale experimentation of embankment reinforced by geosynthetics and rigid piles over soft soil was carried out. Instrumentation was installed for monitoring the evolution of pore water pressure, load transfer toward piles, differential settlements between pile and surrounding soil, lateral displacements at the toe of the slope and geosynthetic strains. From the huge amount of monitoring data of this fullscale experiment, the behaviour of pile and geosynthetic has been studied.

Key words: rigid inclusions, embankment, geosynthetics, instrumentation, reinforcement. 


\section{Introduction}

Le renforcement des sols compressibles par inclusions verticales rigides est une technique courante. Cependant, les quelques méthodes de dimensionnement existantes ne prennent pas en compte toute la complexité des mécanismes développés dans ces ouvrages renforcés (Briançon et al., 2004a) : différents modes de transfert de charge sont proposés, la réaction du sol est rarement prise en compte, le rôle joué par les nappes géosynthétiques disposées dans la plate-forme de transfert de charge n'est pas clairement identifié...

A partir de ce constat, un projet national français ASIRI (Amélioration des Sols par Inclusions RIgides) a été initié pour améliorer les connaissances sur le renforcement par inclusions rigides verticales et proposer des règles de dimensionnement et de mise en œuvre. Dans le cadre de ce projet, une expérimentation en vraie grandeur sous remblai a été réalisée. Quatre plots expérimentaux présentant différentes variantes de renforcement ont été testés sous un remblai de $5 \mathrm{~m}$ de hauteur. Une instrumentation très complète a été mise en place, complétée par des essais de chargement sur inclusions rigides. L'ensemble des mesures a validé l'efficacité du renforcement et a permis d'identifier certains mécanismes de transfert de charge.

\section{2 \\ Site expérimental}

\section{1}

\section{Contexte}

L'expérimentation en vraie grandeur de renforcement de remblais sur sol compressible par inclusions rigides a été conduite par la chaire de géotechnique du Cnam (Conservatoire national des arts et métiers) qui a coordonné les actions, assuré l'instrumentation et le suivi du chantier avec les partenaires du projet national ASIRI.

Cette expérimentation s'est déroulée de juillet 2007 à fin janvier 2008 sur un terrain situé en région parisienne et mis à la disposition du projet par le conseil général de Seine-et-Marne en attente de l'aménagement de la liaison sud de Chelles.

\section{Caractérisation du sol en place}

Différents sondages ont été réalisés pour déterminer l'épaisseur de la couche compressible et la caractériser (Fig. 1) : huit essais pénétrométriques statiques (notés PS sur les figures) et deux sondages carottés (notés SC). Les échantillons prélevés dans ces deux sondages carottés ont été utilisés pour des essais de caractérisation et des essais de compressibilité. La campagne de reconnaissance menée sur le site a permis de définir la succession stratigraphique suivante :

$-1,5 \mathrm{~m}$ de remblai argileux ;

- $1 \mathrm{~m}$ d'argile beige verdâtre ;

$-3,5 \mathrm{~m}$ d'argile peu plastique plus ou moins sableuse ; - entre 2 et 4,5 m d'argile peu plastique sableuse avec des passages d'argile raide ;

- marne et graviers compacts.

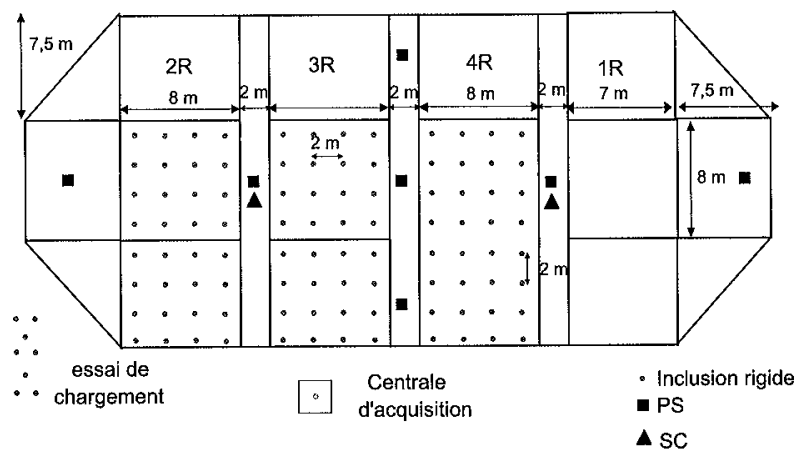

Ho. Implantation des inclusions rigides et des sondages.

View of rigid inclusions and in situ tests.

Le sol en place est homogène, légèrement consolidé $\left(\sigma_{\mathrm{p}}^{\prime}-\sigma_{\mathrm{vo}}^{\prime}\right.$ de l'ordre de 10 à $\left.20 \mathrm{kPa}\right)$ et présente une compressibilité moyenne, le paramètre $C_{c} /\left(1+e_{0}\right)$ variant de 0,1 à 0,25 . Des essais de laboratoire ont permis d'identifier une argile peu plastique plus ou moins sableuse. La position de la nappe a été contrôlée à l'aide d'un piézomètre ; son niveau supérieur se situe à $-2 \mathrm{~m}$. Lépaisseur de la couche compressible, déduite des essais au pénétromètre statique, varie entre 8 et $10,5 \mathrm{~m}$ sous la zone expérimentale (Fig. 2).

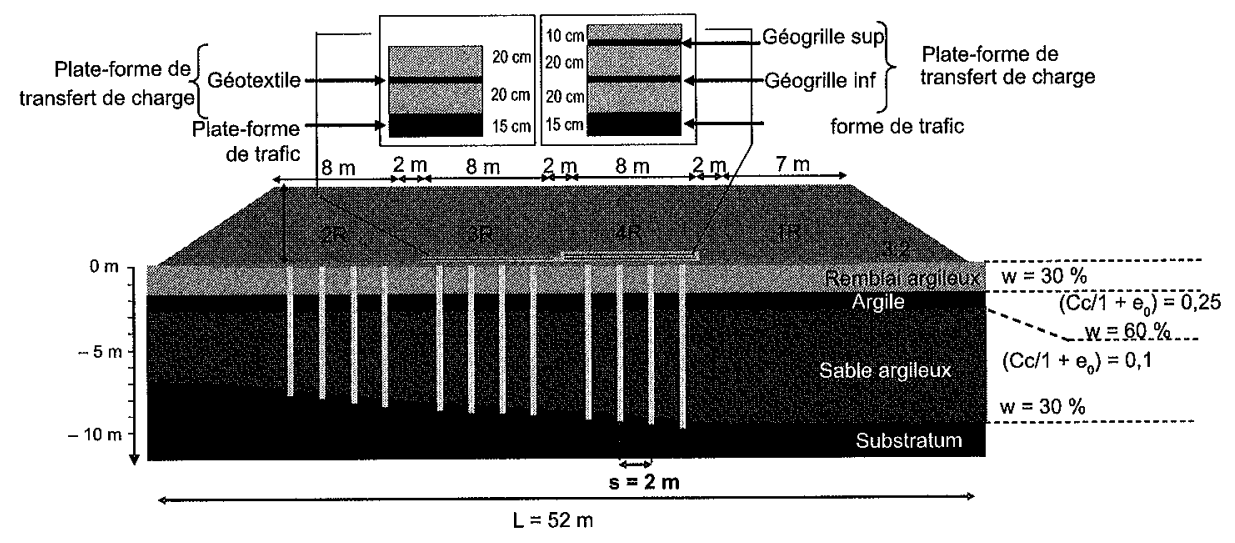




\section{Les plots expérimentaux}

\section{1}

\section{Description des plots expérimentaux}

Quatre remblais expérimentaux ont été mis en œuvre sur ce site (Fig. 2) :

- plot 1R de référence non renforcé par inclusions rigides;

- plot 2R renforcé par inclusions rigides ;

- plot 3R renforcé par inclusions rigides et par une plate-forme de transfert de charge granulaire renforcée par une nappe géotextile ;

- plot 4R renforcé par inclusions rigides et par une plate-forme de transfert de charge granulaire renforcée par deux géogrilles.

Chaque plot est constitué du corps du remblai de $64 \mathrm{~m}^{2}$ de surface et de deux talus de pente $3 \mathrm{H}-2 \mathrm{~V}$. Les quatre plots sont alignés dans un axe nord-est - sudouest. Les plots sont distants de $2 \mathrm{~m}$. Les plots $1 \mathrm{R}$ et 2R disposent d'un troisième talus dans l'axe nord-est - sud-ouest de pente 3H-2V. Seuls les talus nord-ouest des plots sont renforcés par inclusions rigides. Le corps du remblai et les talus nord-ouest sont instrumentés.

Les trois plots renforcés par inclusions rigides contiennent 16 inclusions chacun sous le corps de remblai et 16 inclusions sous le talus renforcé. Les inclusions rigides, de $38 \mathrm{~cm}$ de diamètre, forment un maillage carré avec un taux de recouvrement de 2,8\%.

\section{2}

\section{Réalisation}

Après décapage de la couche végétale, une plateforme de travail de $15 \mathrm{~cm}$ d'épaisseur, en grave roulée 0-70, a été mise en place sur la zone de circulation de la foreuse réalisant les inclusions rigides et sur le plot 1R. La société Keller a réalisé les inclusions rigides refoulantes de $38 \mathrm{~cm}$ de diamètre ; celles-ci ont été recepées à la base de la plate-forme de trafic. Le béton utilisé présente une résistance à la compression égale à $35 \mathrm{MPa}$. Chaque inclusion rigide est ancrée d'une trentaine de centimètre dans la couche de sol dur.

Une plate-forme de transfert de charge a été mise en œuvre sur les plots 3R et 4R. La plate-forme du plot 3R est constituée des $15 \mathrm{~cm}$ du matériau de la plate-forme de trafic, de $20 \mathrm{~cm}$ de grave industrielle primaire $0 / 31,5$ recyclée mixte (déconstruction chaussée et béton) compactée, assimilée B41 dans la classification GTR (noté $G_{\mathrm{F71}}$ ), d'une nappe géotextile TenCate Rock Pec 75/75 et de $20 \mathrm{~cm}$ de grave $G_{\mathrm{F} 71}$. La plate-forme du plot $4 \mathrm{R}$ est constituée des $15 \mathrm{~cm}$ du matériau de la plate-forme de trafic, de $20 \mathrm{~cm}$ de grave $G_{F_{71}}$, d'une géogrille inférieure Miragrid 55/55, de $20 \mathrm{~cm}$ de grave $\mathrm{G}_{\mathrm{F} 71^{\prime}}$ d'une géogrille supérieure Miragrid 55/55 et de $10 \mathrm{~cm}$ de grave $G_{F 71}$.

Le matériau de remblai est un marno-calcaire de classification GTR A1-B5. Le remblai, d'une hauteur totale de $5 \mathrm{~m}$, a été mis en œuvre en trois semaines par couches successives de $30 \mathrm{~cm}$ compactées (fin du remblaiement le 05/09/07 pour le plot 1R, le 12/09/07 pour le plot $4 \mathrm{R}$ et le $19 / 09 / 07$ pour les plots $2 \mathrm{R}$ et $3 \mathrm{R}$ ). Le poids volumique humide de $19 \mathrm{kN} / \mathrm{m}^{3}$ (pour une teneur en eau moyenne de $27,6 \%$ ) a été déterminé in situ au gamma-densimètre.

4

\section{Instrumentation}

Plus de 70 capteurs ont été mis en place sur les quatre plots expérimentaux. Des capteurs de pression totale (notés CPTn sur les figures) positionnés au niveau des têtes d'inclusions, directement sur les inclusions et au droit du sol compressible, dans les inclusions rigides et au-dessus de la plate-forme de transfert de charge ont permis l'analyse des reports de charge. Le tassement du sol compressible a été suivi à l'aide de tassomètres magnétiques en forage (notés TMn) dans les plots 1R et 2R. Le tassement des têtes d'inclusions, du sol à la base du remblai et dans la plate-forme de transfert de charge des plots renforcés par géosynthétiques a été évalué à l'aide de capteurs de pression pour mesure de niveau (notés Tn). A la base du remblai, dans les plots renforcés, ces capteurs sont positionnés de telle sorte que l'on puisse mesurer le tassement différentiel sur la diagonale et sur un côté de la maille centrale. Deux capteurs de pression interstitielle (notés CPIn) suivent ses variations sous le remblai. En pied de talus, les déplacements latéraux ont été mesurés à l'aide de tubes inclinométriques verticaux (IV) et par des capteurs inclinométriques (I) insérés dans les inclusions rigides. La figure 3 illustre l'implantation de l'instrumentation disposée sous la plate-forme de transfert de charge du plot 3R.

Les déformations des divers géosynthétiques de renfort sont mesurées à l'aide de dispositifs à fibres optiques intégrées dans une bande de nappe géosynthétique. Cette technologie, le Geodetect, permet de mesurer avec une grande précision les déformations locales (Briançon et al., 2004b). Deux bandes ont été placées sous le géotextile du plot 3R (Fig. 4) au niveau de la maille centrale du corps du remblai ; une autre installée sous la géogrille inférieure et une dernière sous la géogrille supérieure du plot 4R (Fig. 4).

\section{5}

\section{Mesures}

\section{1}

\section{Résultats du piézomètre et des CPI}

Les deux capteurs de pression interstitielle situés à la même profondeur et dans les plots $1 \mathrm{R}$ et $2 \mathrm{R}$ donnent des résultats similaires en dehors de la phase de mise en place du remblai. Les valeurs de pression interstitielle ramenées en hauteur de nappe équivalente donnent des résultats proches de ceux mesurés par le piézomètre en dehors de la période de mise en œuvre du remblai (Fig. 5).

Lors de la mise en cuvre du remblai, on note une augmentation de la pression interstitielle, plus prononcée dans le plot non renforcé (1R). La surpression est rapidement dissipée dans les deux plots.

La bonne concordance entre les mesures effectuées par les deux capteurs de pression interstitielle et celles relevées sur le piézomètre valide la fiabilité de l'instrumentation et la qualité de sa mise en œuvre, délicate pour ce type de capteurs. 


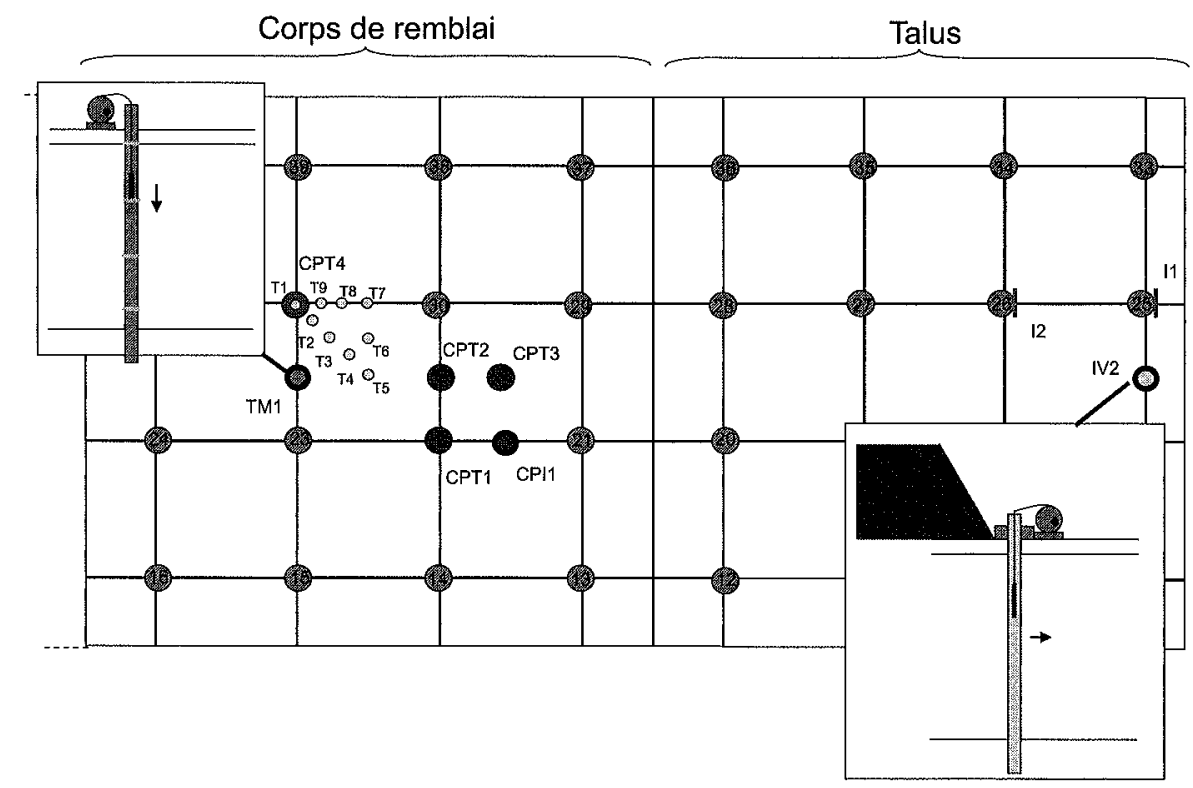

1.6. 0 Implantation des capteurs disposés sous la plate-forme de transfert de charge du plot $3 R$.

Instrumentation of section 3R under the load transfer platform.
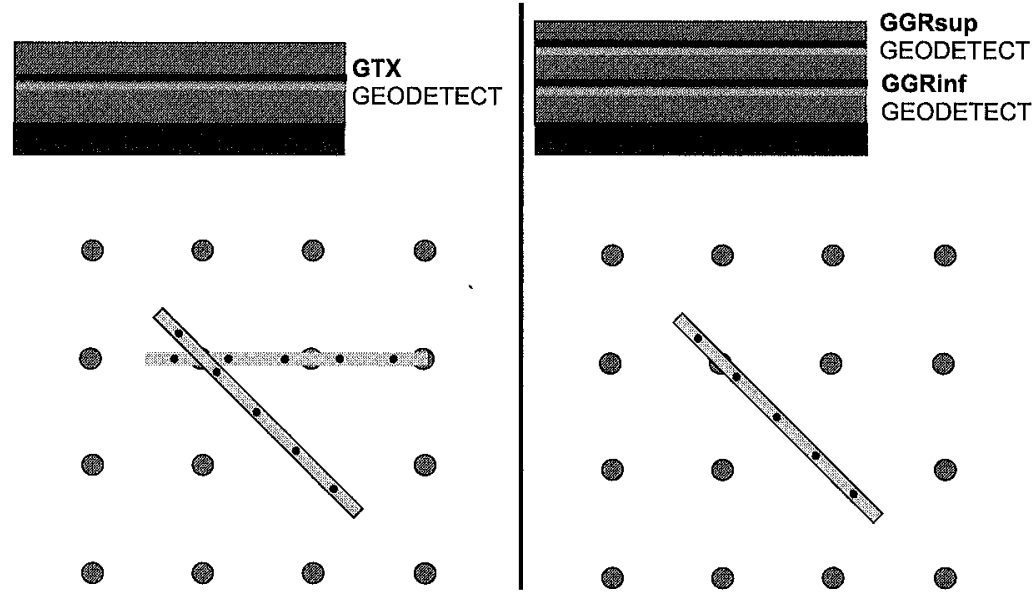

PLOT 3R

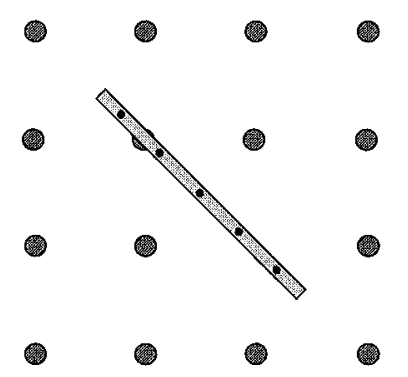

PLOT 4R

16. 4 Implantation des capteurs de déformation des nappes géosynthétiques.

Instrumentation of the geosynthetic sheet strain.

\section{2}

\section{Transfert de charge et efficacité des inclusions}

Le transfert de charge vers les têtes d'inclusions se fait essentiellement pendant la mise en œuvre du remblai puisque $97 \%$ de la charge finale est appliquée sur les têtes en fin de construction. Les deux capteurs positionnés sur le sol à la base du remblai du plot $2 \mathrm{R}$ mesurent lá même contrainte (Fig. 6). Au niveau de la tête d'inclusion, la contrainte mesurée, à savoir $590 \mathrm{kPa}$, est très inférieure à la valeur de $3350 \mathrm{kPa}$, qui représenterait un report total de la charge de la maille sur l'inclusion. Cette contrainte augmente rapidement avec la profondeur puisqu'elle atteint une valeur de $920 \mathrm{kPa}$ à $50 \mathrm{~cm}$ de la tête des inclusions. Cette augmentation de contrainte dans l'inclusion est due au transfert de charge qui s'opère par frottement dans la couche superficielle (remblai argileux) ; les bonnes caractéristiques mécaniques de cette formation semblent permettre une bonne participation du sol support aux mécanismes de transfert de charge. Le faible report de charge sur les têtes d'inclusions peut s'expliquer quant à lui par l'absence de plate-forme de transfert de charge granulaire à la base du remblai. 

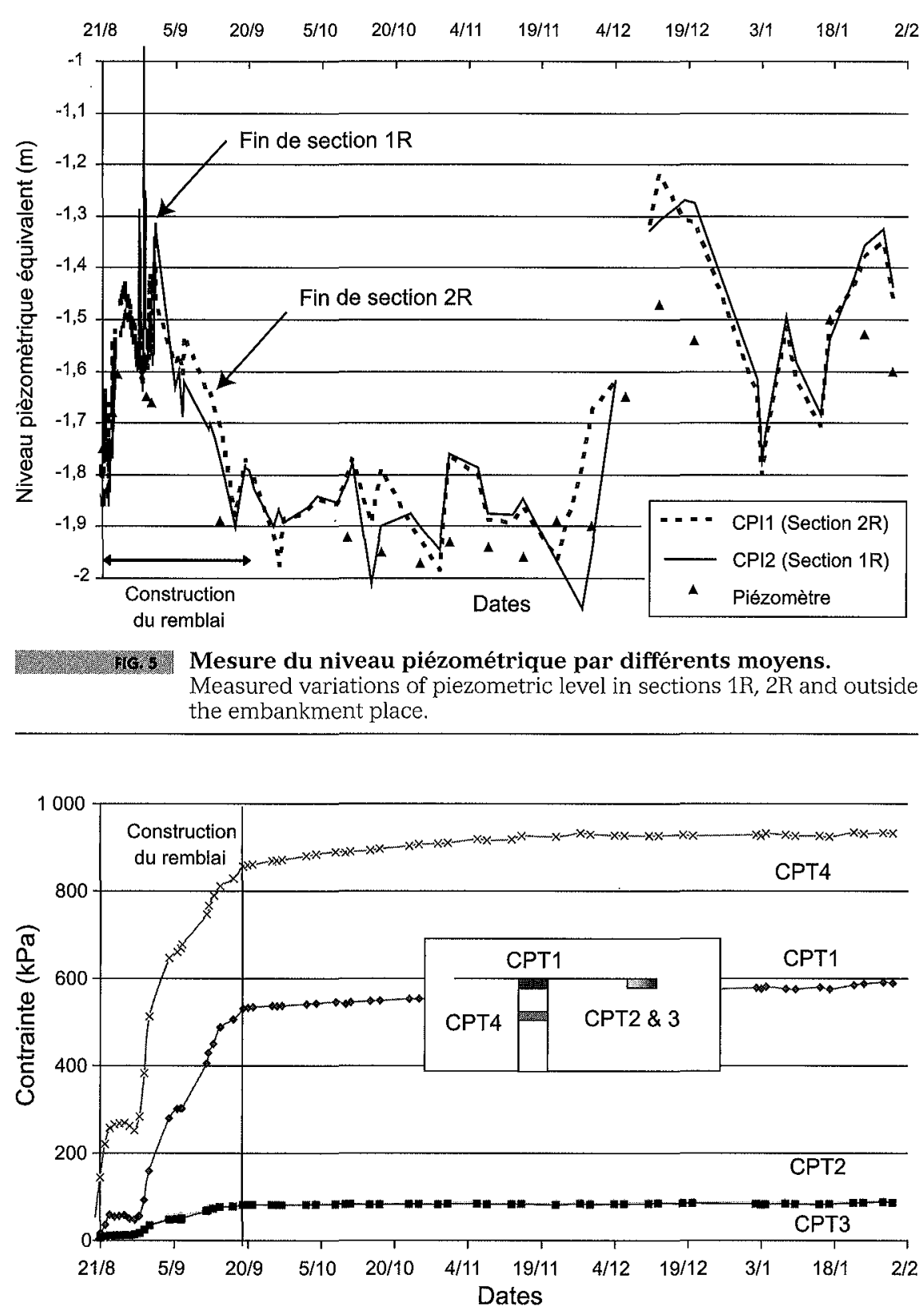

13.

Load transfer in section $2 \mathrm{R}$.

Le transfert de charge vers les têtes d'inclusions est en revanche significativement plus grand dans les plots disposant d'une plate-forme granulaire renforcée par des nappes géosynthétiques puisque la contrainte mesurée sur les têtes d'inclusions atteint des valeurs de l'ordre de $2950 \mathrm{kPa}$ dans le plot 3R (Fig. 7) et $2480 \mathrm{kPa}$ dans le plot 4R (Fig. 8). Pour ces deux plots, la contrainte mesurée sur le sol est négligeable. On note également que les plates-formes de transfert de charge se comportent différemment selon la nature du renforcement géosynthétique. En effet, la contrainte mesurée au droit des têtes d'inclusions au-dessus de la plate-forme de transfert de charge est plus grande dans le plot $3 \mathrm{R}\left(\sigma_{\mathrm{CPT9}}=510 \mathrm{kPa}\right)$ que dans le plot $4 \mathrm{R}$ $\left(\sigma_{\mathrm{CPT} 14}=130 \mathrm{kPa}\right)$. Il est à noter que la valeur mesurée à la base du remblai au droit des inclusions rigides dans le plot $3 \mathrm{R}\left(\sigma_{\mathrm{CPT9}}=510 \mathrm{kPa}\right)$ est comparable à celle mesurée à la base du remblai au droit des inclusions rigides dans le plot $2 \mathrm{R}\left(\sigma_{\mathrm{CPT1}}=590 \mathrm{kPa}\right)$. Dans le plot $3 \mathrm{R}$, la contrainte directement mesurée sur les têtes d'inclusion atteint sa valeur maximale quelques jours après la construction du remblai, puis se stabilise. Dans le plot $4 \mathrm{R}$, il y a redistribution de la contrainte vers le sol puisque la contrainte mesurée par le capteur $\sigma_{\mathrm{CPT10}}$ décroît après avoir atteint sa valeur maximale.

L'efficacité du renforcement a été calculée pour les trois plots (Tableau I). Nous rappelons que l'efficacité E est définie comme le rapport entre la charge réellement appliquée sur une tête d'inclusion et la charge appliquée sur une maille. L'efficacité est maximale si toute la charge appliquée sur la maille est reprise sur la tête d'inclusion.

\section{TABlEAU I Efficacité des renforcements.}

\begin{tabular}{lccc}
\hline & 21 & & $4 R$ \\
\hline Efficacité & $17,9 \%$ & $89,5 \%$ & $74,6 \%$ \\
& $(27,8 \%)^{1}$ & & \\
Taux de transfert de charge à la & $90 \%$ & $86 \%$ & $108 \%$ \\
fin de la construction du remblai & $(92 \%)$ & & \\
\hline
\end{tabular}

(1) Évaluée à $50 \mathrm{~cm}$ sous la tête de l'inclusion. 


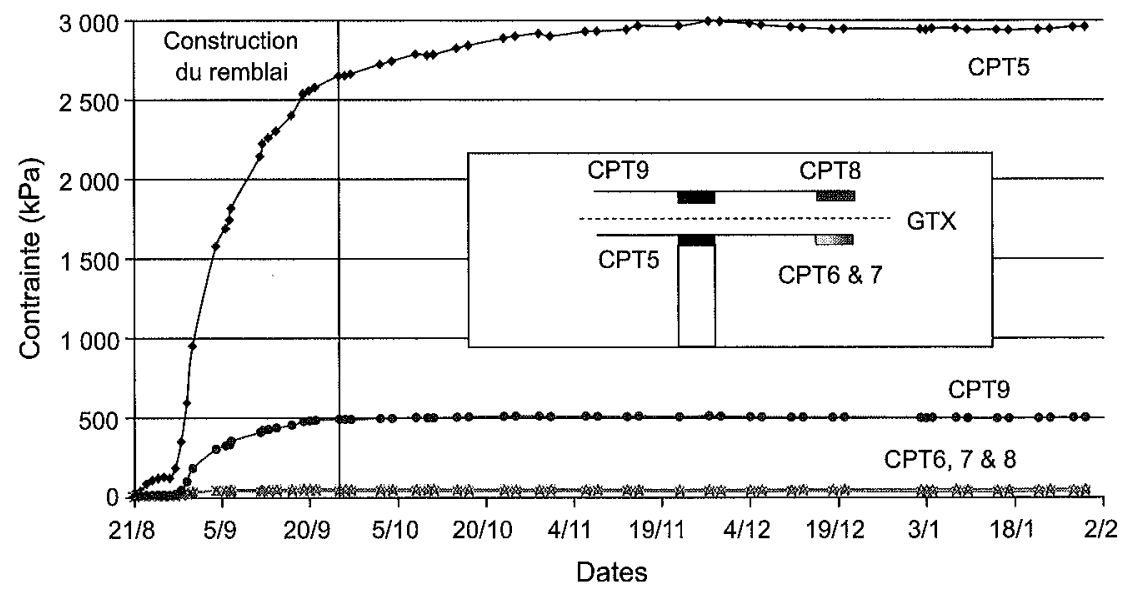

Hesure du transfert de charge pour le plot 3R. Load transfer in section 3R.

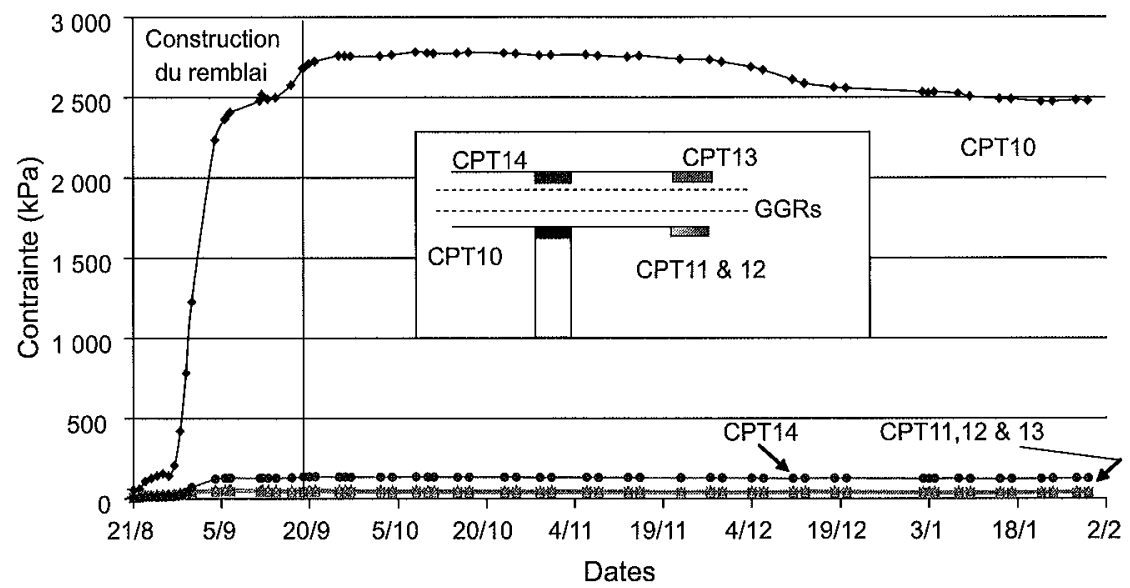

16. 9 Mesure du transfert de charge pour le plot 4R. Load transfer in section $4 \mathrm{R}$.

On peut d'ores et déjà remettre en question la pertinence du paramètre $E$ pour évaluer l'efficacité de ces dispositifs. En effet, ce paramètre a été historiquement défini à la suite d'essais en laboratoire pour lesquels le sol sous-jacent n'était pas pris en compte (Hewlett et Randolph, 1988 ; Low et al., 1994 ; Demerdash, 1996). Or s'il existe une couche de sol de bonne qualité en tête du profil permettant un transfert complémentaire de la charge, l'efficacité sera moindre mais le tassement différentiel sera diminué, ce qui a priori est le but recherché. Il est donc préférable d'évaluer l'efficacité en termes de tassement et non de report de charge mesuré sur les têtes d'inclusions. Le transfert de charge mesuré dans le plot 2R montre que le sol sous-jacent peut participer au transfert de charge par frottement. Il est important de considérer cette couche de sol dans le dimensionnement, car elle est souvent présente soit naturellement (remblai existant à la surface ou croûte superficielle surconsolidée), soit rapportée (plate-forme de trafic). Dans certains cas, la présence de dallettes en béton coiffant les inclusions rigides peut avoir une influence défavorable sur les tassements puisqu'elle diminue ou supprime le frottement sol/inclusion dans la zone où il est le plus important. La présence de dallettes peut donc dans certains cas abaisser le point neutre et solliciter le sol compressible sur une plus grande profondeur. Un calcul simple permet de vérifier si les valeurs de la contrainte sur le sol respectivement mesurée ou calculée à partir de la contrainte mesurée en tête de l'inclusion rigide sont concordantes, si on suppose que la contrainte est uniforme sur le sol. Le tableau II présente cette vérification. Les résultats sont concordants pour le plot 2R. Pour les deux plots disposant d'une plate-forme granulaire, on peut expliquer les écarts par le compactage de la plate-forme au droit des capteurs qui peut fausser les mesures, la mesure de la contrainte verticale sur un sol compressible restant toutefois délicate.

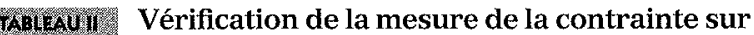
le sol.

\begin{tabular}{|c|c|c|c|}
\hline 110 & $\alpha_{R}$ nes $n$ Pa & 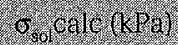 & 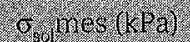 \\
\hline $2 \mathrm{R}$ & 590 & 80 & 87 \\
\hline $3 R$ & 2950 & 13 & 50 \\
\hline $4 \mathrm{R}$ & 2480 & 27 & 36 \\
\hline
\end{tabular}


Les profils de tassements sur la diagonale sont com-

\section{Tassement}

\section{$\sin (x)$}

\section{Tassement du sol compressible}

Les mesures du tassement, au niveau du terrain naturel, par les capteurs de pression de mesure de niveau de liquide et la première bague magnétique des deux tassomètres mettent en évidence une très bonne concordance des mesures (Fig. 9). Le plot non renforcé (1R) subit un tassement de 23 à $26 \mathrm{~cm}$. Le tassement du plot renforcé (2R) est significativement réduit de près de $60 \%$ à la base du remblai. Cette réduction de tassement est aussi observée en profondeur : $70 \%$ à $-2 \mathrm{~m}$ et $80 \%$ à $-5 \mathrm{~m}$. Le renforcement n'accélère pas, dans le cas présent, le temps de tassement qui est déjà très court pour le plot non renforcé.

\section{5.}

\section{Tassement au niveau des têtes d'inclusions}

Les profils de tassement, mesurés à l'aide des capteurs de type (T) au niveau des têtes d'inclusions, sont représentés pour les trois plots à différentes dates (Fig. 10). On peut raisonnablement considérer que le remblai est en construction jusqu'à la date du 19 septembre 2008, même si le phasage de sa mise en cuvre n'a pas permis de rehausser tous les plots de manière simultanée.

Dans le plot 2R, le tassement de la tête d'inclusion est de l'ordre de $8 \mathrm{~mm}$ (Fig. 10). Le tassement du sol présente un profil très homogène jusqu'à $30 \mathrm{~cm}$ de la périphérie de l'inclusion. Dans cette zone proche de l'inclusion, il est difficile de définir le profil exact du tassement car il n'y a qu'un capteur positionné à $10 \mathrm{~cm}$ du fût de l'inclusion. Cependant, le tassement différentiel de deux capteurs consécutifs (Fig. 11, capteurs T3 et T2) met en évidence que le phénomène d'accrochage se développe de manière significative dans l'anneau de sol d'épaisseur $10 \mathrm{~cm}$ entourant l'inclusion.

parables à ceux mesurés sur le côté de la maille.

Les plots disposant d'une plate-forme granulaire ( $3 \mathrm{R}$ et $4 \mathrm{R}$ ) présentent des profils de tassements un peu différents de ceux observés sur le plot 2R. Pour ces plots avec plate-forme, on observe un tassement des inclusions plus élevé $(31 \mathrm{~mm}$ pour $3 \mathrm{R}$ et $28 \mathrm{~mm}$ pour 4R) en adéquation avec les niveaux de contrainte que celles-ci subissent. Dans les deux cas, le tassement mesuré à proximité de l'inclusion rigide est légèrement supérieur à celui mesuré par les capteurs plus éloignés. Le tassement différentiel entre le sol et les inclusions se produit pendant la mise en œuvre du remblai, pour se stabiliser par la suite (Fig. 12). Les plots renforcés réduisent nettement le tassement observé à partir du début de la construction du remblai (Tableau III) ou à partir de la fin de mise en œuvre du remblai (Tableau IV). La comparaison entre les tassements du plot $2 \mathrm{R}$ et ceux des plots $3 R$ et $4 \mathrm{R}$ montre toute l'importance du rôle joué par une plate-forme de transfert de charge. La combinaison de cette plate-forme, qui transfère la charge vers les inclusions, et d'un faible ancrage de ces dernières permet d'obtenir un tassement différentiel moindre au niveau des têtes d'inclusions. Ce tassement différentiel est un paramètre dimensionnant, notamment lorsque le remblai est de faible épaisseur. Bien que l'efficacité $\mathrm{E}$ du plot $3 \mathrm{R}$ soit supérieure à celle du plot 4R, le tassement différentiel est plus faible dans le plot $4 \mathrm{R}$. Cette observation confirme l'observation faite au paragraphe 5.2 sur le manque de pertinence du paramètre E. Le site expérimental présente un tassement après édification du remblai relativement faible ; il est donc nécessaire, lors du dimensionnement, d'imposer un critère de tassement « après mise en œuvre » afin de tirer bénéfice du renforcement. Le renforcement doit à la fois permettre d'obtenir un tassement maîtrisé et stabilisé à la fin de la mise en œuvre de l'ouvrage et être également suffisamment (c souple ) pour éviter des tassements différentiels trop importants au niveau des têtes d'inclusions.

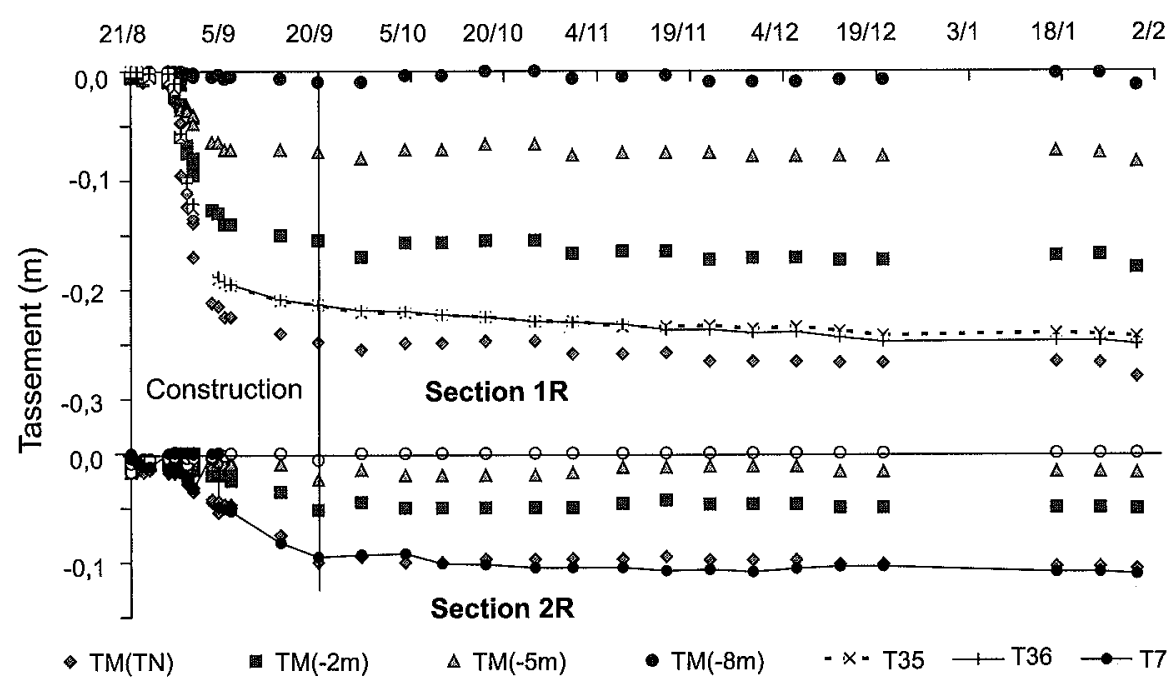

H16. Mesure du tassement du sol compressible pour les plots 1R et 2R. Settlement of soft soil with depth for sections $1 \mathrm{R}$ and 2R. 
Distance à l'axe de l'inclusion rigide $(\mathrm{m})$

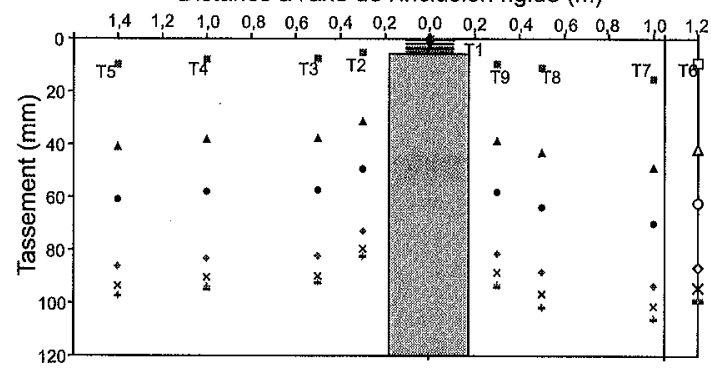

Distance à l'axe de l'inclusion rigide $(\mathrm{m})$

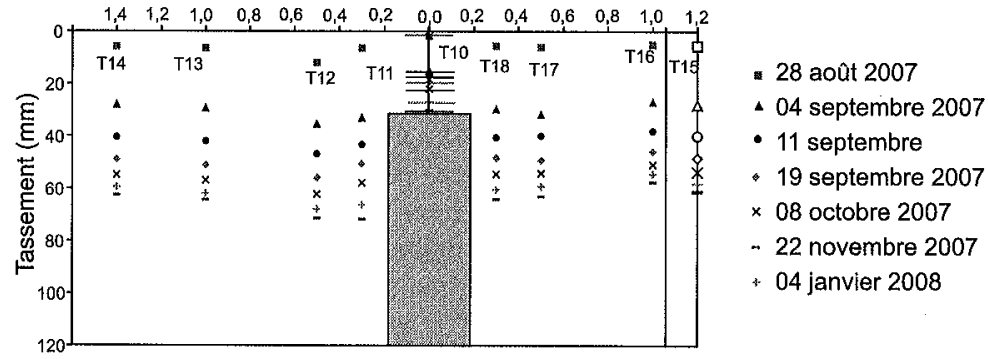

Distance à l'axe de l'inclusion rigide $(\mathrm{m})$

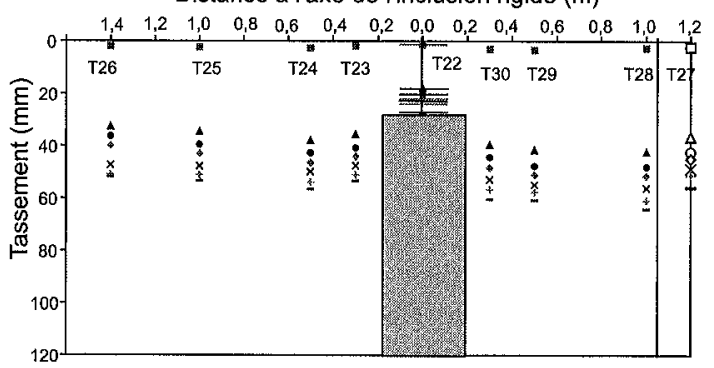

156.10 Mesure du tassement au niveau des têtes d'inclusions pour les plots $2 R$, $3 R$ et $4 R$.

Settlement at the pile level for sections $2 R, 3 R$ and $4 R$.

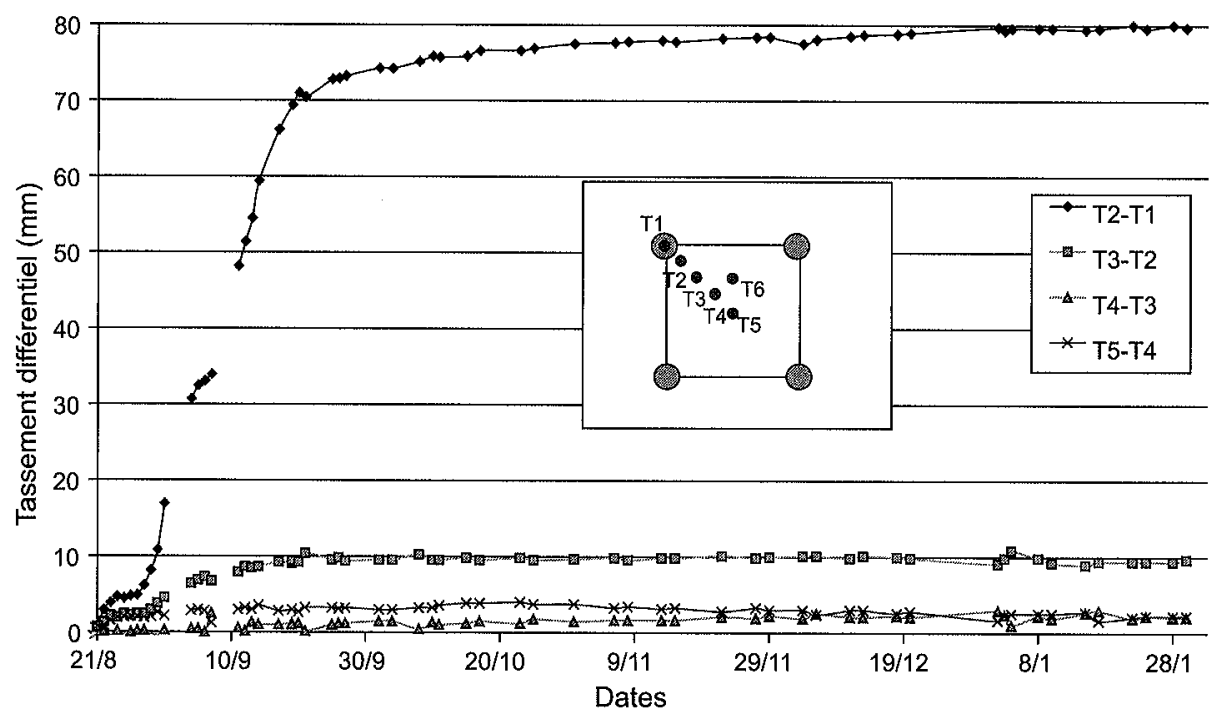

20.11 Tassement différentiel pour le plot 2R. Differential settlement in section $2 \mathrm{R}$.

Tassement compté à partir du début de la construction du remblai.

\begin{tabular}{lcccc}
\hline & 112 & 27 & SR & AR \\
\hline Tassement du sol (mm) & 260 & 105 & 71 & 64 \\
Tassement différentiel sol/IR (mm) & & 97 & 41 & 37
\end{tabular}

TABusoury Tassement compté à partir de la fin de la mise en œuvre du remblai.

\begin{tabular}{lcccc}
\hline & $1 \mathrm{R}$ & $2 \mathrm{R}$ & $3 \mathrm{R}$ & $\mathrm{4R}$ \\
\hline Tassement du sol (mm) & 35 & 18 & 14 & 10 \\
Tassement différentiel sol/IR (mm) & & 15 & 7 & 6
\end{tabular}




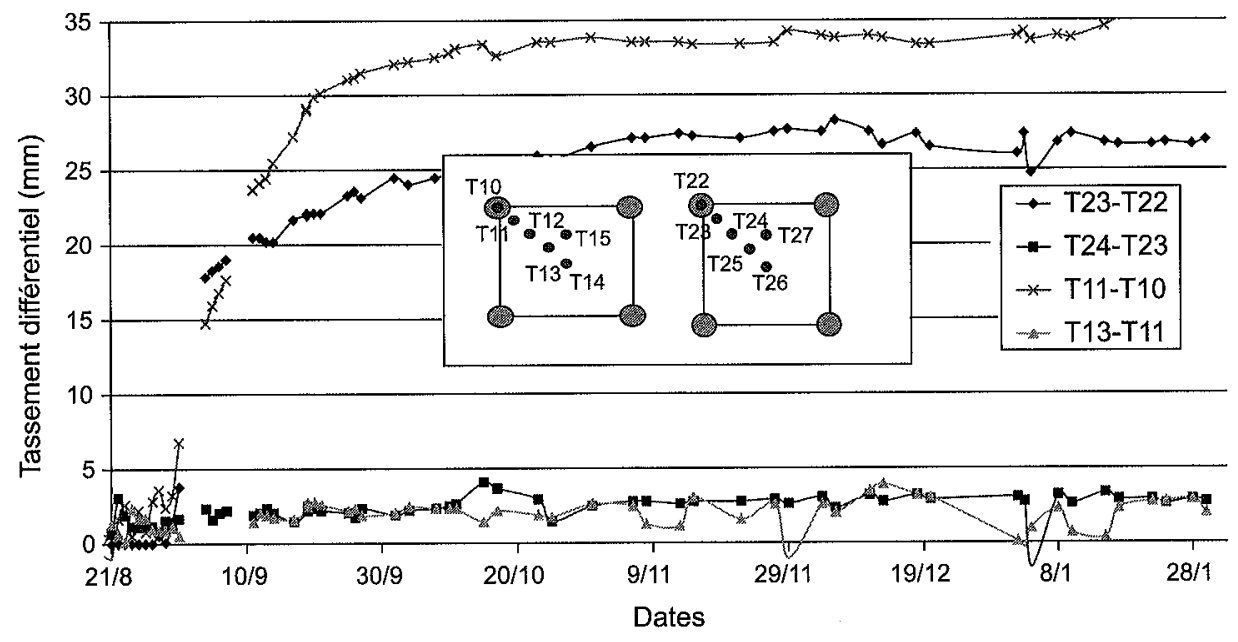

Tassement différentiel pour les plots $3 R$ et $4 R$.

Differential settlement in sections $3 R$ and $4 R$.

\section{4}

\section{Déformation des nappes géosynthétiques}

La figure 13 rassemble les mesures de déformation de la nappe géotextile du plot 3R après la mise en œuvre de la plate-forme de transfert de charge et après la mise en ouvre du remblai. Ces mesures sont effectuées en cinq points sur la diagonale d'une maille (Fig. 13a) et sur le côté d'une maille (Fig. 13b). La symétrie des résultats tend à confirmer une bonne adéquation instrumentation-mesure. Des glissements entre la bande de Geodetect et la nappe géotextile demeurent néanmoins possibles, induisant une déformation éventuellement inférieure à la déformation réelle de la nappe.

On observe que la nappe de géotextile est très peu sollicitée lors du compactage de la plate-forme. A la suite de la mise en œuvre du remblai, celle-ci se déforme principalement au niveau des inclusions rigides, car beaucoup moins sollicitée au droit du sol compressible. Enfin, on note que les déformations sont plus importantes dans le sens des renforts, c'est-à-dire sur le côté de la maille.
La figure 14 présente les mesures de déformation observées sur les deux géogrilles du plot 4R après la mise en œuvre de la plate-forme de transfert de charge et après la mise en œuvre du remblai. Ces mesures sont effectuées en cinq points de la diagonale d'une maille de la géogrille inférieure (Fig. 14a) et supérieure (Fig. 14b). Comme pour le plot 3R, l'instrumentation se révèle bien adaptée à la mesure.

Les géogrilles semblent plus sensibles au compactage que le géotextile. La géogrille inférieure se déforme plus lors de la mise en cuvre de la plateforme granulaire puisqu'elle subit plus de passes de compactage. Lors de la mise en cuvre du remblai, on constate que les géogrilles se déforment principalement à proximité des inclusions et qu'elles ne sont pas du tout sollicitées entre celles-ci.

La comparaison des mesures de déformation montrent que les nappes géosynthétiques n'ont pas le même comportement. La nappe géotextile travaille en ( membrane ) lors de la mise en œuvre du remblai et se déforme dans une zone plus large autour des inclusions rigides. Les géogrilles sont pré-tendues lors du

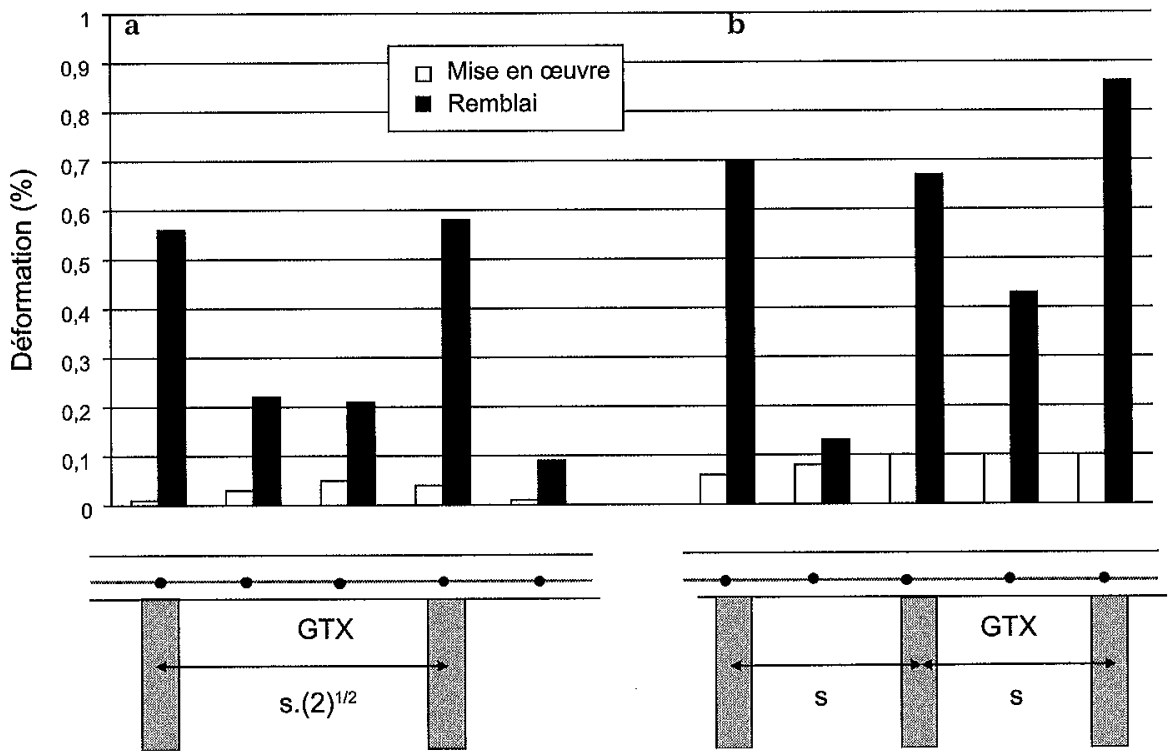

Déformation du géotextile pour le plot 3R.

Geotextile strain in section 3R. 


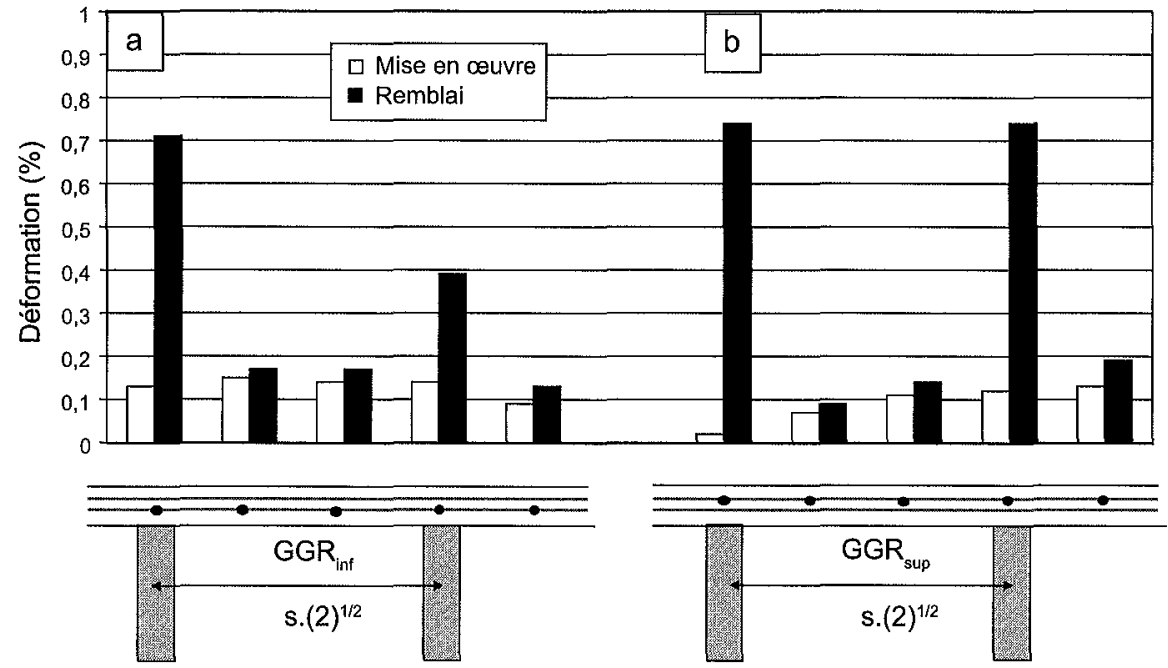

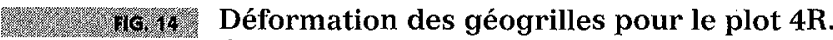

Geogrids strain in section 4R.

compactage de sorte à former un complexe granulaire renforcé qui transfère la charge vers les têtes d'inclusions lors de la mise en œuvre du remblai. Là encore, les nappes travaillent principalement dans une zone plus large que les inclusions rigides.

\section{6}

\section{Tassement dans les zones de recourrement}

L'écart de comportement entre nappes de géosynthétiques de nature différente est aussi mis en lumière par l'examen du tassement sous les zones de recouvrement (Fig. 15). Celui-ci, dans le cas des nappes de géotextiles, n'est affecté ni par le compactage ni par le remblaiement de sorte que le tassement est identique sous les zones de recouvrement et en partie courante des nappes. Ce n'est pas le cas pour les géogrilles, où on observe une augmentation de tassement sous les zones de recouvrement notamment lors de la phase de mise en œuvre. Le compactage de la plate-forme a, semble-t-il, (c ouvert » les zones de recouvrement des nappes de géogrilles.
56

\section{Déplacements latéraux en pied de talus et inclinaison des inclusions rigides}

L'inclinaison mesurée en tête des inclusions rigides en pied de talus est quasiment nulle. Le déplacement latéral en pied de talus, mesuré sur le plot $1 \mathrm{R}$, est de l'ordre de $30 \mathrm{~mm}$. Ce déplacement est diminué de plus de moitié dans les plots renforcés. Ces valeurs étant relativement faibles, il est difficile d'attribuer une influence significative à l'interposition des nappes de géosynthétiques. Le rapport $\Gamma$ entre le déplacement latéral maximal et le tassement total dans l'axe du remblai, calculé pour le plot non renforcé, est égal à 0,12 ; du même ordre de grandeur que les valeurs préconisées dans le fascicule $62-V$ du CCTG $(\Gamma=0,16$ en pied de talus pour des pentes de talus entre 0,5 et 0,67 ).

On observe enfin que les déplacements latéraux sont maximaux dans la couche d'argile située à $1,5 \mathrm{~m}$ de profondeur, identifiée comme la plus compressible.

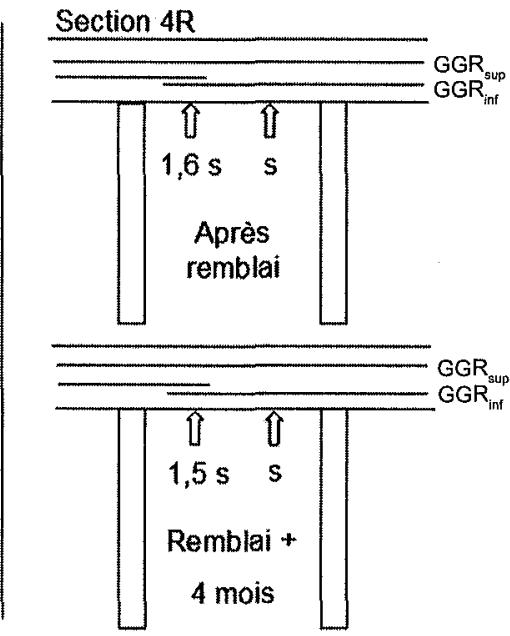

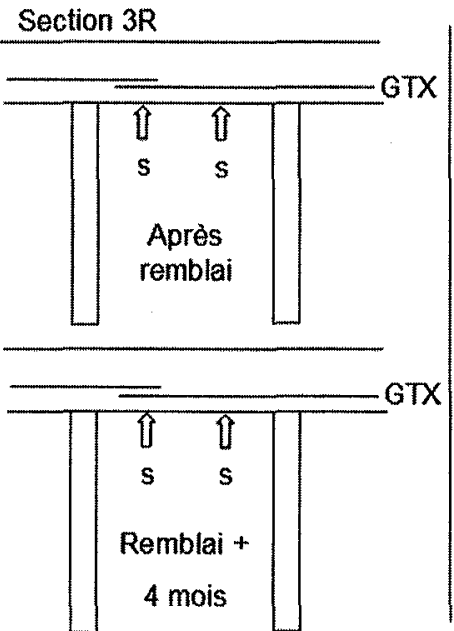

16. 15. Tassement mesuré sous les zones de recouvrement des nappes géosynthétiques.

Settlement under the overlapping areas of geosynthetics. 


\section{6}

Analyse

\section{1.}

\section{Identification des mécanismes}

Le transfert de charge s'effectue par une combinaison de mécanismes dépendants de la présence ou non de certains éléments de renforcement.

En l'absence d'une plate-forme de transfert de charge, la concentration des contraintes verticales vers les têtes d'inclusions se fait à la base du remblai. Les mécanismes associés ne permettent pas d'obtenir une efficacité importante au niveau des têtes puisque seulement un sixième du ( poids de la maille ) est repris par l'inclusion ; le sol support est donc assez fortement chargé. Un second mode de transfert de charge vers les inclusions s'opère par frottement du sol au contact des inclusions. Ce transfert est d'autant plus important que la couche de surface est de bonne qualité. Les mesures de tassement du sol compressible (Fig. 9) montrent que le tassement est fortement diminué à $2 \mathrm{~m}$ de profondeur, conduisant à une estimation de la position du ( point neutre » entre 2 et $5 \mathrm{~m}$ de profondeur.

Ce transfert de charge nécessite un déplacement important du sol au niveau des têtes et donc un tassement différentiel sol/inclusion inacceptable dans le cas d'un remblai de faible épaisseur. Dans le cas présent (remblai d'une épaisseur de $5 \mathrm{~m}$ ), on peut estimer qu'un plan (c d'égal tassement ) a pu se développer au sein du remblai même si aucun dispositif de mesure n'a été malheureusement installé pour le confirmer.

La présence d'une plate-forme de transfert de charge améliore très nettement ce transfert vers les inclusions. Aucun plot associé à une plate-forme granulaire dépourvue de géosynthétique n'ayant été réalisé dans le cadre de cette expérimentation, il n'est ainsi pas possible au stade actuel de séparer les influences respectives de la plate-forme et des nappes de géosynthétiques.

Les mesures de déformation des nappes (Figs. 13 et 14), de tassement sous les zones de recouvrement (Fig. 15), les mesures de contrainte au-dessus de la plate-forme (Figs. 7 et 8) montrent que, pour des tassements comparables, les mécanismes de transfert de charge sont différents selon la nature des nappes géosynthétiques.

Dans le cas du plot 3R, la nappe de géotextiles se met en tension pendant la mise en œuvre du remblai et travaille essentiellement dans une zone périphérique à l'inclusion rigide. Cette mise en tension a tendance à confiner le sol situé autour de l'inclusion, générant des tassements plus importants en périphérie immédiate de celle-ci. L'inclusion très chargée et peu ancrée tasse mais ne transfère qu'une très faible part de sa charge au sol avoisinant, qui tasse dans les mêmes proportions autant puisque l'équilibre s'établit lorsque l'inclusion peut reprendre l'ensemble des efforts transmis (pointe et frottement positif sur sa partie inférieure). Au-dessus de la plate-forme de transfert de charge, on observe une répartition de contrainte analogue à celle observée en base du plot $2 \mathrm{R}$ : tout se passe comme si des inclusions fictives prolongeaient les inclusions réelles du plot 3R sur la hauteur de la plate-forme de transfert de charge.
Dans le cas du plot $4 \mathrm{R}$, les géogrilles sont mises en tension dès le compactage de la plate-forme de transfert de charge. Cette pré-tension (dont l'effet s'avère analogue à celui d'une pré-contrainte dans une dalle) améliore le transfert de charge sur la hauteur de la plate-forme qui se trouve associé à un tassement différentiel plus faible. Ceci est bien reflété par la faible différence observée au toit de la plate-forme, entre les contraintes mesurées au droit des inclusions et celles mesurées entre (au droit du sol). Lors de la construction du remblai, les géogrilles travaillent exclusivement à la périphérie des inclusions rigides. L'inclusion étant très chargée et peu ancrée tasse et redistribue une part de sa charge au sol avoisinant placé plus en profondeur.

En première analyse, il n'est pas aisé d'expliquer les différences entre les mécanismes de transfert de charge observées dans les plots 3R et 4R. En effet, pour un tassement comparable, la charge reprise par les inclusions du plot 3R est nettement supérieure à l'effort repris par celles du plot 4R. Cette différence ne s'explique pas par l'ancrage des inclusions, identique dans les deux configurations. Une analyse plus complète, avec une modélisation numérique des nappes, permettra de vérifier si la différence de transfert de charge observée peut être expliquée par la nature des géosynthétiques. Peu d'expérimentations en vraie grandeur permettent une analyse pertinente de ces mécanismes ; on citera cependant celles d'Almeida et al. (2007) ou Liu et al. (2007) qui amènent à des observations semblables aux nôtres, en particulier sur

- l'influence de la réaction du sol compressible sur le tassement différentiel notamment lorsque la couche superficielle est de bonne qualité (Almeida et al., 2007) ;

- le transfert de charge vers les inclusions rigides via une plate-forme granulaire renforcée et la répartition des tensions dans les nappes géosynthétiques (Liu et al., 2007).

\section{2}

\section{Corrélation avec les essais de chargement}

La figure 16 présente, pour les trois plots renforcés, les tassements mesurés en tête des inclusions en fonction de la charge mesurée, au même stade, immédiatement sous les têtes. Ces éléments peuvent être comparés à la courbe déduite de l'essai de chargement réalisé par le Laboratoire central des ponts et chaussées (LCPC) sur une inclusion isolée, obtenue en rapportant le tassement mesuré en tête à la valeur de la contrainte mesurée en pointe (Fig. 16).

Sur la courbe déduite de l'essai de chargement, on distingue plusieurs segments :

- AB : application d'une charge de $150 \mathrm{kN}$;

- BC : fluage pendant 30 minutes sous $150 \mathrm{kN}$;

- CD : application d'une charge de $300 \mathrm{kN}$;

- DE : fluage pendant 30 minutes sous $300 \mathrm{kN}$;

- EF : application d'une charge de $450 \mathrm{kN}$;

- FG : fluage pendant 30 minutes sous $450 \mathrm{kN}$;

- GH : application d'une charge de $600 \mathrm{kN}$;

- HI : fluage pendant 30 minutes sous $600 \mathrm{kN}$.

Malgré le faible nombre de paliers de chargement et l'absence de mesures en continu du tassement durant le passage d'un palier de charge au suivant, on constate un bon accord entre les deux séries de 


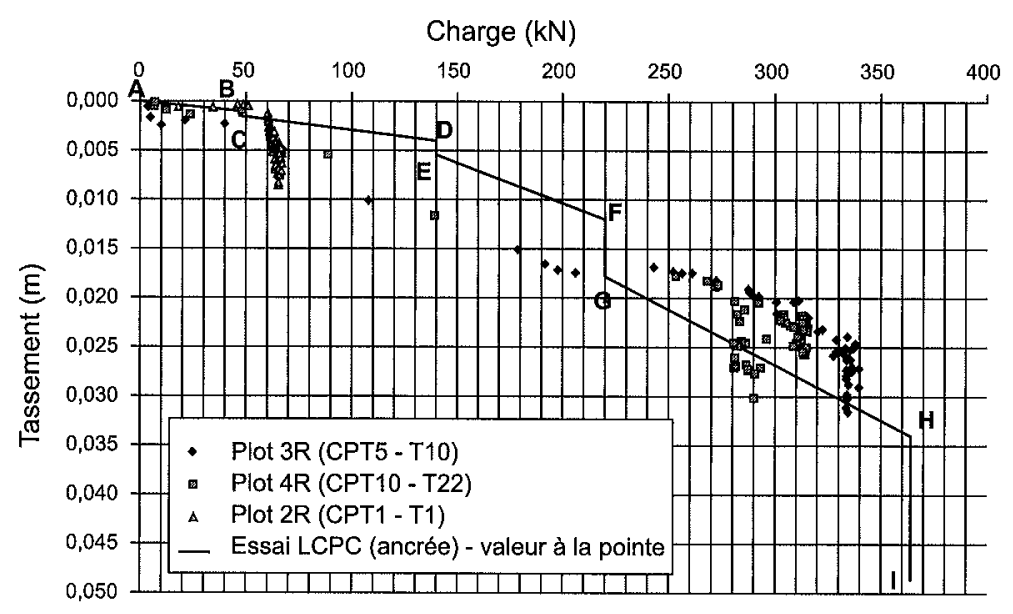

nc 16 Comparaison entre les charges mesurées en tête des inclusions et les valeurs de pointe de l'essai de chargement statique pour les plots $2 R$, 3R et $4 R$.

Comparison between the loads measured on pile head and the end bearing capacity of the static loading test in sections $2 \mathrm{R}, 3 \mathrm{R}$ and $4 \mathrm{R}$.

mesures : les tassements mesurés sous les plots en tête des inclusions sont comparables, à niveau de charge identique, à ceux mesurés en pointe lors du chargement de l'inclusion isolée. Ce constat tend à montrer que les inclusions placées sous les plots expérimentaux mobilisent essentiellement leur résistance en pointe et seulement une part très faible de frottement positif audessus de ce niveau. Le faible ancrage des inclusions sous les niveaux compressibles du site explique, de manière plausible, ce comportement.

\section{7 \\ Conclusion}

Dans le cadre du projet national ASIRI, une expérimentation en vraie grandeur sous remblai renforcé par inclusions rigides a été réalisée. L'ensemble des mesures a permis d'acquérir des données expérimentales de tout premier ordre concernant le comportement des remblais sur ce type de renforcement. Un soin tout particulier a été apporté au choix de l'instrumentation et à la mise en œuvre des plots expérimentaux.

Divers mécanismes de transfert de charge ont pu être identifiés. Les mesures ont permis de vérifier l'influence de la réaction du sol sous la plate-forme granulaire, mettant en défaut les hypothèses conservatives de certaines méthodes de dimensionnement actuelles.
L'influence de la présence d'une plate-forme de transfert de charge a été démontrée en termes de concentration des efforts vers les inclusions; cependant, dans le cas présent, le dispositif semble aussi ( efficace ) malgré son absence, en termes de tassement. Lorsque le sol en place, combiné à la plate-forme de travail, présente une couche superficielle susceptible d'assurer un transfert de charge par frottement, l'efficacité exprimée par rapport aux contraintes déterminées sur les têtes d'inclusions n'est pas forcément représentative de l'efficacité (c réelle » du dispositif. Les mesures montrent enfin que pour des tassements comparables, les mécanismes de transfert de charge semblent être différents selon la nature des nappes géosynthétiques renforçant les plates-formes granulaires.

Cette base de données constitue un acquis important du projet national ASIRI, au cœur des actions qui se poursuivent pour confronter et valider des méthodes de dimensionnement représentatives du comportement réel de ces ouvrages.

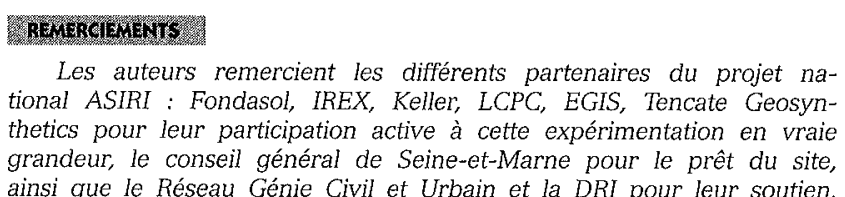

\section{Bibliographie}

Almeida M.S.S., Ehrlich M., Spotti A.P., Marques M.E.S. - Embankment supported on piles with biaxial geogrids. Geotechnical Engineering, 160 (GE4), 2007, p. 185-192.

Briançon L., Kastner R., Simon B., Dias D. - État des connaissances - Amélioration des sols par inclusions rigides. Proc. of International Symposium on Ground Improvement (ASEP-GI 2004), 9-10 septembre 2004, Presses des Ponts, 2004a, p. $15-43$.
Briançon L., Nancey A., Caquel F., Villard P. - New technology for strain measurements in soil and the survey of reinforced earth constructions. Proc. of EUROGEO 3, March 1-3 2004, Munich, Germany, 2004b, p. 471-476.

Demerdash M.A. - An experimental study of piled embankments incorporating geosynthetic basal reinforcement. Thesis, University of Newcastle Upon Tyne, Department of civil Engineering, 1996, p. 196.
Hewlett W.J., Randolph M.F. - Analysis of piled embankments. Ground Engineering, 21 (3), 1988, p. 12-18.

Liu H.L., Ng C.W.W., Fei K. - Performance of a Geogrid-Reinforced and Pile-Supported Highway Embankment over Soft Clay : Case Study. Journal of Geotechnical and Geoenvironnemental Engineering, 133 (12), 2007, p. 1483-1493.

Low B.K., Tang S.K., Choa V. - Arching in piled embankments. Journal of Geotechnical and Geoenvironnemental Engineering, 120 (11), 1994, p. 191-1937. 